\title{
Defective Self-Renewal and Differentiation of GBA-Deficient Neural Stem Cells Can Be Restored By Macrophage Colony-Stimulating Factor
}

\author{
Hyun Lee $e^{1,2}$, Jae-sung Bae ${ }^{1,3,4,}$, and Hee Kyung $\mathrm{Jin}^{1,2, *}$
}

Gaucher disease (GD) is an autosomal recessive lysosomal storage disorder caused by mutations in the glucocerebrosidase gene (GBA), which encodes the lysosomal enzyme glucosylceramidase (GCase). Deficiency in GCase leads to characteristic visceral pathology and lethal neurological manifestations in some patients. Investigations into neurogenesis have suggested that neurodegenerative disorders, such as GD, could be overcome or at least ameliorated by the generation of new neurons. Bone marrowderived mesenchymal stem cells (BM-MSCs) are potential candidates for use in the treatment of neurodegenerative disorders because of their ability to promote neurogenesis. Our objective was to examine the mechanism of neurogenesis by BM-MSCs in GD. We found that neural stem cells (NSCs) derived from a neuronopathic GD model exhibited decreased ability for self-renewal and neuronal differentiation. Co-culture of GBA-deficient NSCs with BM-MSCs resulted in an enhanced capacity for self-renewal, and an increased ability for differentiation into neurons or oligodendrocytes. Enhanced proliferation and neuronal differentiation of GBA-deficient NSCs was associated with elevated release of macrophage colony-stimulating factor (M-CSF) from BM-MSCs. Our findings suggest that soluble M-CSF derived from BM-MSCs can modulate GBA-deficient NSCs, resulting in their improved proliferation and neuronal differentiation.

\footnotetext{
${ }^{1}$ Stem Cell Neuroplasticity Research Group, ${ }^{2}$ Department of Laboratory Animal Medicine, Cell and Matrix Research Institute, College of Veterinary Medicine, Kyungpook National University, Daegu 702-701, Korea, ${ }^{3}$ Department of Physiology, Cell and Matrix Research Institute, School of Medicine, ${ }^{4}$ Department of Biomedical Science, BK21 Plus KNU Biomedical Convergence Program, Kyungpook National University, Daegu 700842 , Korea

*Correspondence: hkjin@knu.ac.kr (HKJ); jsbae@knu.ac.kr (JSB)
}

Received 4 May, 2015; revised 6 June, 2015; accepted 18 June, 2015; published online 18 August, 2015

Keywords: BM-MSC, gaucher disease, M-CSF, neural stem cells, neurogenesis

\section{INTRODUCTION}

Gaucher disease (GD) is caused by an inherited deficiency in the enzyme glucosylceramidase (GCase). Mutations in the glucocerebrosidase gene (GBA), which encodes GCase, leads to defective hydrolysis of glucosylceramide (GluCer), subsequently resulting in the storage of GluCer in the liver, spleen, bone marrow, and central nervous system (CNS) (Beutler and Grabowski, 2001; Brady et al., 1966). Clinically, two major categories of GD have been recognized: non-neuronopathic and neuronopathic. These can be further sub-divided into three types. Type $1 \mathrm{GD}$ is the most common form of the disease and includes non-neuronopathic variants. Types 2 and $3 \mathrm{GD}$ encompass acute and subacute neuronopathic variants (Beutler and Grabowski, 2001). In neuronopathic GD, pathogenesis in the CNS is related to neuronal death and dropout, which is propagated by the toxic effects of GluCer (Conradi et al., 1984; 1988; Sidransky et al., 2004). Little is known about the molecular events leading to neuronal death, and at present, there is no treatment available for neuronopathic GD.

In the adult brain, neurogenesis of neural stem cells (NSCs) and progenitor cells occurs in two regions: the subventricular zone of the lateral ventricles and the subgranular zone of the hippocampus (Ming and Song, 2011). The discovery of neurogenesis in the adult brain has raised the possibility of potential therapeutic applications to overcome neurodegenerative diseases such as Parkinson's disease, Huntington's disease, and Alzheimer's disease (Ming and Song, 2011). These findings indicate that neurodegenerative diseases, such as GD, are suitable targets for the therapeutic stimulation and regulation of neurogenesis.

In a previous study, we showed that bone marrow-derived mesenchymal stem cells (BM-MSCs) contributed to improving neurogenesis in a mouse model of Niemann-Pick type C (Lee et al., 2013). This therapeutic function of MSCs can, in part, be explained by their production of diffusible trophic factors (Block et al., 2009; Ren et al., 2007; Zhang et al., 2004; 2005). Deng et al. (2006) previously demonstrated elicitation of neurogenesis and promotion of functional recovery by transplanting MSCs in rhesus monkeys. Our goal in the current study was to investigate the neurogenic potential of BM-MSCs, and of soluble factors released from BM-MSCs, for the promotion of NSC neurogenesis in a neuronopathic GD model.

Treatment with BM-MSCs resulted in an enhanced capacity for self-renewal, proliferation, and neuronal differentiation of 
GBA-deficient NSCs. Our observations suggest that macrophage colony-stimulating factor (M-CSF) plays a key role as a paracrine factor for the neurogenic effects of BM-MSCs in GBA-deficient NSCs.

\section{MATERIALS AND METHODS}

\section{Animals}

$G b a^{\text {flox/lox; }}$,nestin-Cre mice were used as a model of neuronpathic GD (Enquist et al., 2007). Gba $a^{\text {floxflox }}$ mice were crossed with $\mathrm{Gba}^{\text {floxt+}}$;nestin-Cre mice to generate $G b a^{\text {floxflox }}$;nestin-Cre mice (hereafter referred to as $\mathrm{Gba}^{-/-}$mice) and $\mathrm{Gba}^{\text {flox/+}}$;nestinCre mice (hereafter referred to as $\mathrm{Gba}^{+-}$mice), which served as wild-type controls. Genotyping was performed by polymerase chain reaction (PCR), using genomic DNA extracted from the tails of mice (Farfel-Becker et al., 2009). All procedures were performed in accordance with a protocol approved by the Kyungpook National University Institutional Animal Care and Use Committee. Animals were housed in a temperaturecontrolled room with a 12-h light-dark cycle.

\section{Isolation and culture of NSCs}

Using a previously described method (Chen et al., 2007) with some minor modifications, dissociated NSCs were prepared from the cerebral cortex of 1-day-old neonatal $\mathrm{Gba}^{+/-}$or $\mathrm{Gba}^{-/-}$mice. The cortex was removed and kept in ice-cold $\mathrm{Ca}^{2+} / \mathrm{Mg}^{2+}$-free Hank's balanced salt solution (Invitrogen, USA). The cortex was then mechanically dissociated in NSC culture medium [Dulbecco's modified Eagle's medium (DMEM)/F12 (Invitrogen), 1\% N2 supplement (Invitrogen), $20 \mathrm{ng} / \mathrm{ml}$ epidermal growth factor (Peprotech, USA), $20 \mathrm{ng} / \mathrm{ml}$ basic fibroblast growth factor (Peprotech)]. Cell suspensions were filtered through a $40-\mu \mathrm{m}$ cell strainer (BD Biosciences, USA) and seeded into individual wells of a six-well tissue culture plates $\left(2 \times 10^{5}\right.$ cells/well; BD Biosciences). NSCs proliferated in suspension and formed aggregates that are known as neurospheres (NSs). Every second days, half the medium in each well was replaced with fresh culture medium.

\section{Isolation and culture of BM-MSCs}

With the aid of a 40- $\mu \mathrm{m}$ cell strainer, single-cell suspensions were obtained from bone marrow that was harvested from tibias and femurs. These were dissected from 4- to 6-week-old wild-type mice. Based on a previously published protocol (Bae et al., 2007), approximately $1 \times 10^{6}$ cells were plated in $25-\mathrm{cm}^{2}$ flasks containing MesenCult MSC Basal Medium and Mesenchymal Stem Cell Stimulatory Supplements (Stem Cell Technologies, Canada). Cell cultures were grown for 1 week; cells that adhered to the culture surface were designated as BM-MSCs and were used in subsequent experiments.

\section{Enzyme activity assays}

Cells were lysed in homogenization buffer [50 mM HEPES (Invitrogen), 150 mM NaCl (Sigma), 0.2\% Igepal CA-630 (Sigma), protease inhibitor (Calbiochem, USA)]. GCase activities were determined by the catalysis of (7-nitro-2-1,3-benzoxadiazol4-yl)-o-erythro (NBD)-C12-GluCer (Avanti Polar Lipids, USA) to NBD-C12-ceramide, as described previously (Motabar et al., 2012), with some modifications using an ultraperformance liquid chromatography (UPLC) system (Waters, USA). Quantitation was achieved by comparing to NBD-ceramide (Avanti Polar Lipids) standards.

\section{Lipid extraction and GluCer quantitation}

We prepared samples for lipid extraction as previously de- scribed (He et al., 2010). To quantify GluCer levels, human recombinant acid ceramidase (rhAC) and GCase (Cerezyme, Genzyme Corporation, USA) were added to the lipid extracts, and GluCer was fully hydrolyzed to sphingosine. The amount of sphingosine was then quantified using the procedure described by He et al. (2005). Briefly, $2 \mu \mathrm{l}$ of lipid extract in $0.2 \%$ Igepal CA-630 was mixed with $2 \mu \mathrm{l}$ of a GluCer hydrolysis buffer $(0.2$ $\mathrm{M}$ citrate-phosphate buffer, $\mathrm{pH} 4.5,0.3 \mathrm{M} \mathrm{NaCl}, 50 \mathrm{ng} / \mu \mathrm{l}$ rhAC, $2.5 \%$ GCase) and incubated at $37^{\circ} \mathrm{C}$ for $1 \mathrm{~h}$. Reactions were stopped by adding $20 \mu \mathrm{l}$ of NDA derivatization reaction mixture (25 mM borate buffer, pH 9.0, 2.5 mM NDA, 2.5 mM sodium cyanide). The reaction mixture was diluted 1:3 with ethanol, incubated at $50^{\circ} \mathrm{C}$ for $10 \mathrm{~min}$, and then centrifuged $(13,000 \times g$ for $5 \mathrm{~min})$. An aliquot $(30 \mu \mathrm{l})$ of the supernatant was then transferred to a glass sample vial and $5 \mu$ was applied onto an UPLC system for analysis. Fluorescent sphingosine derivatives were monitored using a model 474 scanning fluorescence detector (Waters). Quantitation of the sphingosine peak was calculated from sphingosine standard calibration curves, using the Waters Millennium software. To calculate the final GluCer content of samples, background of endogenous sphingosine and ceramide signals, determined as above, using a reaction mixture lacking GCase, were subtracted from the signal obtained in the presence of rhAC and GCase.

\section{Co-culture of BM-MSCs and NSCs}

For indirect three-dimensional co-culture experiments, Millicell Hanging Cell Culture Inserts (Millipore, USA) with a pore size of $1.0 \mu \mathrm{m}$ were placed on top of previously plated NSCs. BMMSCs were seeded onto the insert at a density of $3 \times 10^{4}$ cells per insert. In this system, there was no direct contact between NSCs and BM-MSCs. During the 7-day long incubation period, half the medium was replenished with fresh medium every $72 \mathrm{~h}$.

\section{NS formation assays}

To examine the effects of BM-MSCs on NS formation, NSs were mechanically dissociated, and the resulting viable cells quantified. These cells $\left(1 \times 10^{4}\right.$ cells/well in uncoated 24 -well plates; BD Biosciences) were also used to assess the selfrenewal ability in NSC culture medium. Following co-culture with BM-MSCs or treatment with recombinant murine M-CSF (2-50 ng/ml; R\&D Systems, USA), newly formed NSs were counted in each well using an IX71 microscope (Olympus Co., Japan). A minimum cutoff diameter of $50 \mu \mathrm{m}$ was used to define NSs.

\section{NSC proliferation assays}

The proliferative activity of NSCs was evaluated by 5-bromo-2deoxyuridine (BrdU) immunocytochemistry. Single-cell suspensions from NSs were seeded on glass coverslips coated with poly-L-ornithine (Sigma, USA) and laminin (Invitrogen), at a density of $1 \times 10^{4}$ cells $/ \mathrm{cm}^{2}$. Cells were incubated with BMMSCs or M-CSF for 7 days, labeled with a $10 \mu \mathrm{M}$ of BrdU (Sigma), and incubated for an additional $12 \mathrm{~h}$. After the labeling medium was removed, cells were fixed with phosphatebuffered $4 \%(\mathrm{w} / \mathrm{v})$ paraformaldehyde (Sigma) for $20 \mathrm{~min}$ at room temperature (RT). To denature nuclear DNA, cells were incubated in $2 \mathrm{~N} \mathrm{HCl}$ for $1 \mathrm{~h}$, and then in $0.15 \mathrm{M}$ sodium borate for $15 \mathrm{~min}$ (all from Sigma). Cells were washed with phosphatebuffered saline (PBS; Invitrogen), processed for immunofluorescence analysis of $\mathrm{BrdU}$, and incubated with the nuclear counterstain 4',6-diamidino-2-phenylindole (DAPI; Vector Laboratories Inc., USA). 


\section{NSC differentiation assays}

For NSC differentiation assays, single-cell suspensions cultured in vitro for 7 days were plated on glass coverslips coated with poly-L-ornithine and laminin at a density of $1 \times 10^{4}$ cells $/ \mathrm{cm}^{2}$. Coverslips were incubated in Neurobasal-A medium supplemented with $100 \mathrm{U} / \mathrm{ml}$ penicillin/streptomycin, $2 \mathrm{mM} \mathrm{L-glutamine,}$ $10 \mu \mathrm{g} / \mathrm{ml}$ of heparin, $2 \%$ B-27 supplement, and $3 \%$ fetal bovine serum (FBS; all from Invitrogen). Differentiated cultures were processed for immunofluorescence staining at 7 days postplating.

\section{Immunocytochemistry}

Cells were fixed with $0.1 \mathrm{M}$ PBS containing $4 \%$ paraformaldehyde at RT for $15 \mathrm{~min}$, and then permeabilized with $0.1 \%$ Triton X-100 (Sigma) in PBS for 5 min. Cells were preincubated for 10 min with $3 \%$ normal goat serum (Vector Laboratories Inc.) and 2\% bovine serum albumin (BSA; Invitrogen) in PBS containing $0.4 \%$ Triton $\mathrm{X}-100$ to block background signals. For the NSC differentiation assays, differentiated cultures were incubated with various primary antibodies overnight at $4^{\circ} \mathrm{C}$. We used a mouse monoclonal antibody against $\beta$ III-tubulin (diluted 1:400; Chemicon, USA) as a marker for neurons, a rabbit polyclonal antibody against GFAP (diluted 1:1,000; Dako, Denmark) as a marker for astrocytes, or a rabbit polyclonal antibody against MBP (diluted 1:500; Abcam, UK) as a marker for oligodendrocytes. For visualization of primary antibodies, appropriate Alexa Fluor 488-conjugated secondary antibodies (diluted 1:1,000; Molecular Probes, USA) were added to cultures and allowed to incubate for $1 \mathrm{~h}$ at RT. Immunofluorescence in cells was analyzed using a laser-scanning confocal microscope equipped with FluoView SV1000 imaging software (Olympus FV1000, Olympus Co., Japan), or with a BX51 microscope (Olympus Co.).

\section{Neurite outgrowth assay}

Morphological analysis of differentiated neurons was recorded using the MetaMorph software (Universal Imaging Corp., USA). All recordings and MetaMorph analysis were performed in a blinded manner.

\section{Antibody-based mouse cytokine arrays}

RayBio Mouse custom Cytokine Antibody Arrays (Raybiotech, USA) were used to assay cell culture supernatants from coculture experiments, according to the manufacturer's instructions. Membranes were incubated with $2 \mathrm{ml}$ of blocking buffer at RT for $30 \mathrm{~min}$. About $1 \mathrm{ml}$ of the conditioned medium from samples with or without co-cultured BM-MSCs was then added, followed by incubation overnight at $4^{\circ} \mathrm{C}$. After decanting samples, membranes were washed with wash buffers I and II at RT with shaking at $120 \mathrm{rpm}$. Membranes were then incubated with $1 \mathrm{ml}$ of biotin-conjugated antibodies $(1: 250)$ overnight at $4^{\circ} \mathrm{C}$ and washed, followed by incubation with $2 \mathrm{ml}$ of horseradish peroxidase-conjugated streptavidin (1:1000) for $1 \mathrm{~h}$ at RT, and further washing. Detection buffers $C$ and $D$ were used for visualization of spots. Membranes were wrapped in plastic wrap, exposed to Kodak X-Omat radiographic film (Kodak, USA) for $20 \mathrm{~min}$, and signals were detected using a film developer. Each film was scanned into an image processor, and densitometric measurements were performed using an imaging densitometer (Bio-Rad, USA), followed by quantitation with Bio-Rad analysis software. Densitometry and statistical analyses were performed on the immunoblots, with normalization to the positive and negative controls on the array.
Reverse-transcription PCR (RT-PCR) and quantitative PCR (qPCR) assays

The RNeasy Plus Mini Kit (Qiagen, Germany) was used to extract RNA from cell lysates. We synthesized complementary DNA (cDNA) from $5 \mu \mathrm{g}$ of total RNA, using a cDNA Synthesis Kit (Clontech, USA), according to the manufacturer's instructions. Reactions were incubated at $42^{\circ} \mathrm{C}$ for $1 \mathrm{~h}$, and then at $70^{\circ} \mathrm{C}$ for $10 \mathrm{~min}$ to arrest CDNA synthesis. We performed qPCR assays using a Corbett research RG-6000 thermal cycler (Corbett Life Science, Australia). The thermal cycling profile involved 40 amplification cycles $\left(95^{\circ} \mathrm{C}\right.$ for $10 \mathrm{~s}, 58^{\circ} \mathrm{C}$ for $15 \mathrm{~s}, 72^{\circ} \mathrm{C}$ for $20 \mathrm{~s})$. We used oligonucleotide primers that were specific for Mcsf (5'-AGT CTG TCT TCC ACC TGC TG-3' and 5'-TTC CAC CTG TCT GTC CTC AT-3'), IL-1ra (5'-GCA GCA CAG GCT GGT GAA TGA C-3' and $5^{\prime}$-TGC CCC CGT GGA TGC CCA AG-3'), MCP-1 (5'-ATG CAG TTA ATG CCC CAC TC-3' and 5'TTC CTT ATT GGG GTC AGC AC-3'), Mmp2 (5'-CCC CGA TGC TGA TAC TGA-3' and 5'-CTG TCC GCC AAA TAA ACC$\left.3^{\prime}\right)$, Mmp3 (5'-TAG AAG GAG GCA GCA GAG AA-3' and 5'GGG TAG GAT GAG CAC ACA AC-3') and GAPDH (5'-AAC AGC CTC AAG ATC ATC AGC-3' and 5'-TTG GCA GGT TTT TCT AGA CGG $\left.-3^{\prime}\right)$.

\section{Enzyme-linked immunosorbent assay (ELISA)}

determination of M-CSF concentrations

We used a Quantikine Mouse M-CSF ELISA Kit (R\&D Systems) and approved M-CSF standards to measure the concentration of M-CSF, according to the manufacturer's instructions. Each standard and experimental sample was assayed in duplicate, and the results were averaged.

\section{Statistical analysis}

Comparisons between two groups were conducted with Student's $t$-test. Tukey's HSD test and repeated measures analysis of variance test (ANOVA) were used for multigroup comparisons, with the aid of SPSS statistical software. Differences were accepted to be statistically significant at $p<0.05$.

\section{RESULTS}

Deletion of Gba results in severe GCase deficiency and substrate accumulation of GluCer in NSCs

To confirm that nestin-Cre-mediated deletion of the GCase gene disrupted GCase activity, NSCs were obtained from $\mathrm{Gba}^{+/}$and $\mathrm{Gba}^{-/-}$mice. NSCs isolated from the cortex of $\mathrm{Gba}^{-/-}$ neonatal mouse exhibited reduced GCase activity (Fig. 1A), and higher levels of GluCer compared with that in control NSCs (Fig. 1B).

BM-MSCs enhance the self-renewal and proliferation of $\mathrm{Gba}^{-/} \mathrm{NSCs}$

To examine the neurogenic potential of BM-MSCs, we used self-renewal and proliferation assays (Fig. 2A). The number of $\mathrm{NSs}$ in $\mathrm{Gba}^{-\gamma}$ cultures was significantly lower compared with that in $\mathrm{Gba}^{+/}$cultures (Fig. 2B). In addition, indirect co-culture of $\mathrm{Gba}^{-/}$NSCs with BM-MSCs resulted in the increased formation of NSs (Fig. 2B). BM-MSCs also induced an increase in NS formation in $\mathrm{Gba}^{+-}$cultures. To assess proliferation, adherent NSCs were pulsed with BrdU and the proportion of labeled cells analyzed (Fig. 2C). BM-MSCs exhibited increased BrdU incorporation in $\mathrm{Gba}^{+/}$and $\mathrm{Gba}^{-/}$cultures in comparison with untreated control cultures (Fig. $2 \mathrm{C}$ ). These results indicate that BM-MSCs promote NSC proliferation. To validate whether the effect of BM-MSCs was mediated by GCase activity, we ana- 

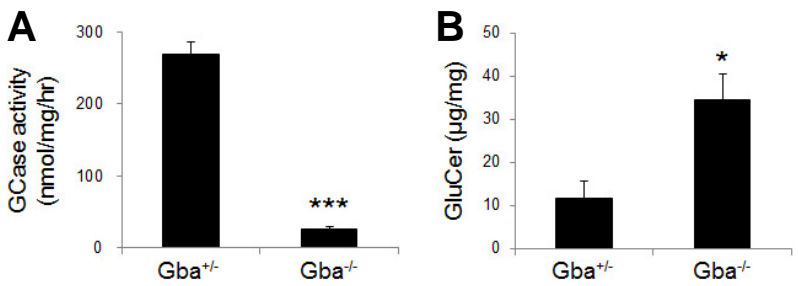

Fig. 1. Deletion of Gba results in a severe enzyme deficiency and accumulation of GluCer in the NSCs. (A) GCase activity was reduced to a greater degree in NSCs of $\mathrm{Gba}^{-/-}$mice compared with that in NSCs of $\mathrm{Gba}^{+-}$mice ( $n=5$ per group). (B) GluCer accumulated in NSCs from $\mathrm{Gba}^{-/}$mice ( $n=5$ per group). All data are presented as the mean \pm SEM. ${ }^{*} p<0.05$ compared with the $\mathrm{Gba}^{+-}$ controls.

lyzed its activity in NSCs co-cultured with BM-MSCs. Activities of GCase in $\mathrm{Gba}^{-/}$and $\mathrm{Gba}^{+/}$cells co-cultured with NSCs were similar to those in cells that were not co-cultured with BMMSCs (Fig. 2D). Our results suggest that BM-MSCs increase self-renewal and proliferation of NSCs. Although the effects of BM-MSCs were not limited to conditions where GCase activity was knocked down, our findings indicate that BM-MSCs promote self-renewal and proliferation of $\mathrm{Gba}^{-/} \mathrm{NSCs}$. In addition, these results provide evidence that the effects of BM-MSCs can be attributed to the indirect effect of neurogenic soluble factors secreted from BM-MSCs.

\section{BM-MSCs affect differentiation of NSCs into specific cell types}

In addition to their ability for self-renewal, the second hallmark of stemness in NSCs is multilineage differentiation (Reynolds and Weiss, 1996). We dissociated NSs and co-cultured cells with BM-MSCs in differentiation media. Immunocytochemistry was performed to investigate the effects of BM-MSCs on Gba NSC cultures and their capacity for differentiation into the three main neural lineages (Fig. 3A). Expression of three neural lineage markers ( $\beta$ III-tubulin, MBP and GFAP) were detected in NSC-derived cells regardless of whether they were co-cultured with BM-MSCs (Figs. 3B-3E). In comparison with $\mathrm{Gba}^{+/-}$cells, the efficiency of neuronal differentiation was not significantly different. We observed signifi- cantly reduced neurite outgrowth and number of processes in differentiated neurons from $\mathrm{Gba}^{-/} \mathrm{NSC}$ cultures (Figs. 3B and 3C). Indirect co-culture of $\mathrm{Gba}^{-/}$NSCs with BMMSCs resulted in significantly enhanced efficiency of neuronal differentiation, increased neurite outgrowth, and an increased number of processes (Fig. 3C). Compared with $\mathrm{Gba}^{+/} \mathrm{NSCs}$, we observed a reduced rate of differentiation for MBPexpressing cells in $\mathrm{Gba}^{-/}$NSC cultures (Fig. 3D). Co-culture with BM-MSCs resulted in a significant increase in the number of differentiating oligodendro- cytes in $\mathrm{Gba}^{-/}$NSC cultures (Fig. 3D). Compared with $\mathrm{Gba}^{+/}$cells however, there were no significant changes in the numbers of GFAP-positive cells in differentiated $\mathrm{Gba}^{-/}$NSC cultures (Fig. 3E). Overall, our results indicate that BM-MSCs selectively promote neurogenesis, neuronal morphogenesis, and oligo- genesis of NSCs.

\section{BM-MSCs increase neurotrophic factor signaling}

Our observations indicate that soluble bioactive factors secreted from BM-MSCs could promote the proliferation of $\mathrm{Gba}^{-/}$ NSCs. To identify these factors, we screened and compared the conditioned media of the various $\mathrm{Gba}^{+/}$and $\mathrm{Gba}^{-/-} \mathrm{NSCs}$ cultures for 50 different secreted cytokines, using an antibodybased mouse cytokine array (Fig. 4A). Expression levels of $\mathrm{M}$ CSF, IL-1ra, MCP-1, MMP-2 and MMP-3 were greater in the conditioned media of $\mathrm{Gba}^{--}$NSCs co-cultured with BM-MSCs than in the conditioned media of NSCs without co-culture (Fig. $4 \mathrm{~A})$. These results suggest the involvement of M-CSF, IL-1ra, MCP-1, MMP-2, and MMP-3 with respect to the self-renewal of $\mathrm{Gba}^{--}$NSCs co-cultured with BM-MSCs. Using qPCR assays, we examined mRNA expression levels of Mcsf, IL-1ra, MCP-1, $\mathrm{Mmp2}$, and $\mathrm{Mmp} 3$ in $\mathrm{Gba}^{+/-}$and $\mathrm{Gba}^{-/} \mathrm{NSCs}$. Compared with controls, expression of Mcsf was significantly decreased in $\mathrm{Gba}^{-/}$NSCs (Fig. 4B). Levels of IL-1ra, MCP-1, Mmp2, and $\mathrm{Mmp3}$ were increased in $\mathrm{Gba}^{-/} \mathrm{NSC}$ compared with those in $\mathrm{Gba}^{+/}$NSCs. We suspect that reduced expression of M-CSF might lead to the impaired self-renewal observed in $\mathrm{Gba}^{-/}$ NSCs. M-CSF derived from BM-MSCs is a potential candidate molecule for alleviating this defect. To confirm the secretion of this factor, we performed ELISAs and found that M-CSF levels were significantly elevated in the conditioned media of $\mathrm{Gba}^{-/}$ NSCs co-cultured with BM-MSCs (Fig. 4C). We also found that M-CSF levels were significantly decreased in $\mathrm{Gba}^{--}$NSCs compared with those in $\mathrm{Gba}^{+-}$NSCs (Fig. 4C), neither of which were co-cultured with BM-MSCs.

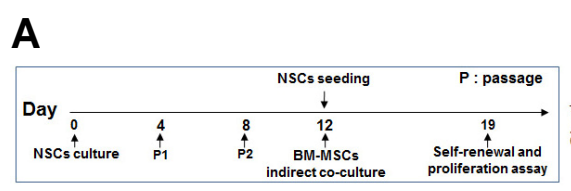

C

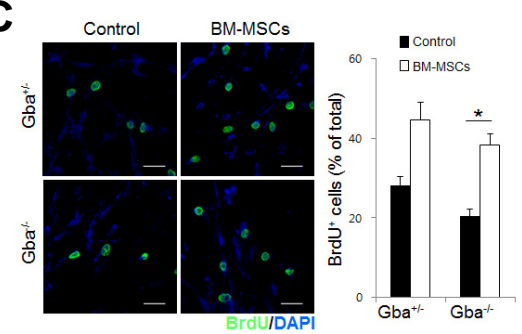

B
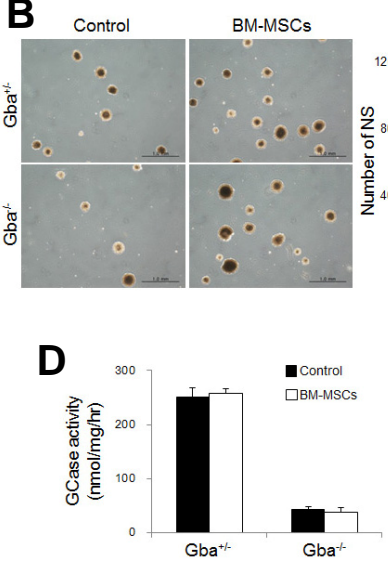

Fig. 2. BM-MSCs enhance the self-renewal and proliferation of $\mathrm{Gba}^{-/}$NSCs. (A) Experimental design to determine the effects of BM-MSCs on $\mathrm{Gba}^{-/}$NSCs. (B) Light micrographs of NSs following BM-MSC co-culture with $\mathrm{Gba}^{-\alpha}$ NSCs (scale bar, $1 \mathrm{~mm}$ ). Selfrenewal capacity is expressed as the number of NSs ( $n=6$ per group). (C) Effect of BM-MSCs on the proliferation of $\mathrm{Gba}^{-1-}$ NSCs (scale bar, $20 \mu \mathrm{m}$ ). Proliferation ability was assessed by the percentage of BrdUpositive cells ( $n=7$ per group). (D) Effect of BM-MSCs on the GCase activity of $\mathrm{Gba}^{--}$ NSCs ( $n=3$ per group). All data are presented as the mean \pm SEM. ${ }^{*} p<0.05,{ }^{* *} p<$ $0.01,{ }^{* * *} p<0.005$. 

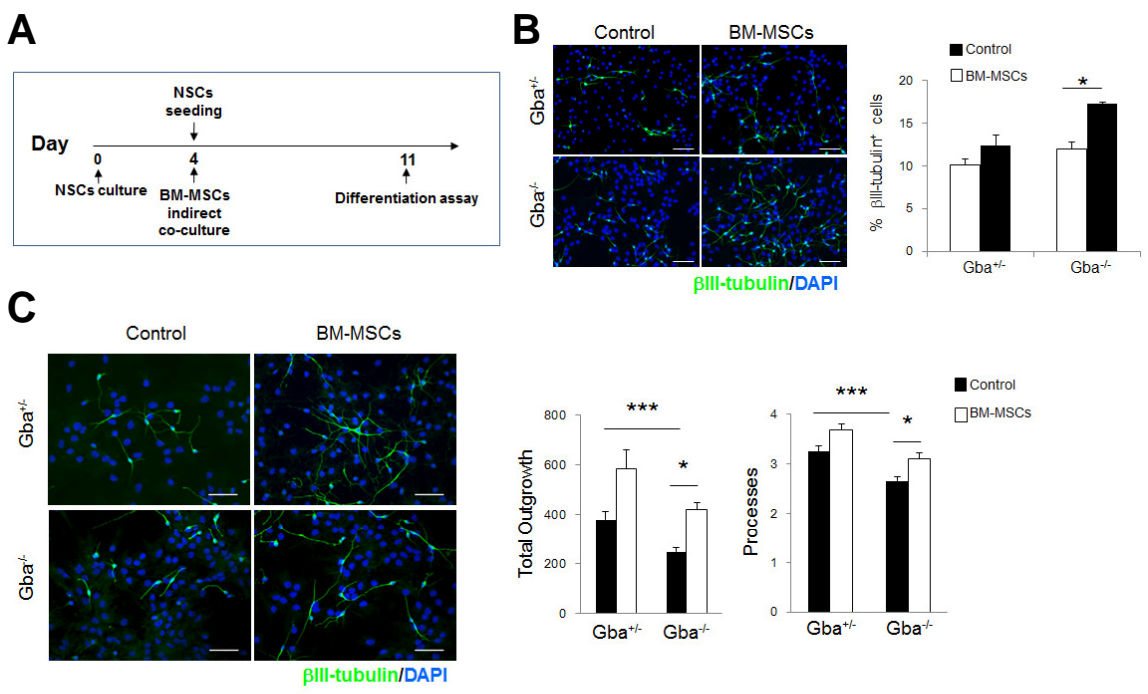

D

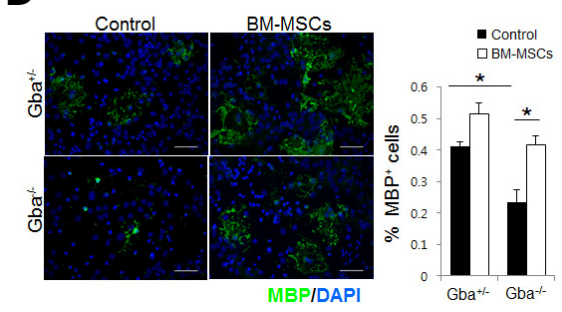

E

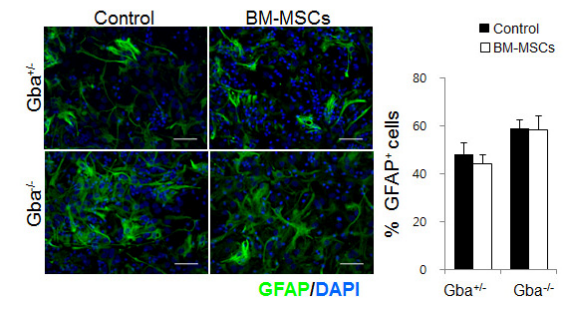

Fig. 3. Indirect co-culture with BM-MSCs results in enhanced differentiation of Gba $^{-\alpha}$ NSCs into specific cell types. (A) Differentiation of NSCs was induced for 7 days, with or without BM-MSC co-culture, using an indirect three-dimensional system. Cells were stained with specific cell type markers. Representative fluorescence images and quantitative analysis of $\beta$ III-tubulin/DAPI (B, C), MBP/DAPI (D), and GFAP/DAPI $(E)$ are shown (scale bar, $50 \mu \mathrm{m}, n=5$ per group). Data are presented as the mean percentage of immunopositive cells to DAPI-stained cells for each group. (C) Total outgrowth of differentiated neuron dendrites and the number of dendritic processes are shown. For each measurement, at least 60 cells per disc were counted from randomly selected fields of view $(n=5$ discs from different mouse origins). All data are presented as the mean \pm SEM. ${ }^{*} p<0.05,{ }^{* *} p<0.005$.

\section{M-CSF enhances the self-renewal, proliferation, and neuronal differentiation of $\mathrm{Gba}^{-/}$NSCs}

To examine the neurogenic potential of M-CSF, we evaluated the effects of recombinant murine M-CSF on the self-renewal of NSs at different concentrations. M-CSF increased the number of NSs in $\mathrm{Gba}^{-/}$cultures at 10 and $50 \mathrm{ng} / \mathrm{ml}$ (Fig. 5A). Therefore, we used $10 \mathrm{ng} / \mathrm{ml}$ M-CSF for subsequent experiments. Compared with $\mathrm{Gba}^{+-} \mathrm{NSs}$, the self-renewal ability of $\mathrm{Gba}^{-/} \mathrm{NSs}$ was significantly decreased. The $\mathrm{Gba}^{-/} \mathrm{NSs}$ treated with MCSF exhibited increased formation of NSs (Fig. 5A). To assess proliferation, the percentage of BrdU-labeled cells was determined. M-CSF increased BrdU incorporation in $\mathrm{Gba}^{-/-}$cultures relative to that in untreated $\mathrm{Gba}^{--}$cultures (Fig. 5B). In addition to examining the effects of M-CSF on neuronal differentiation, NSs were dissociated and treated with M-CSF in differentiation media. After 7 days, the expression of neuronal markers was evaluated in NSC-derived cells. Compared with $\mathrm{Gba}^{+/}$cells, $\mathrm{Gba}^{-/-}$neurons showed significantly decreased neuronal outgrowth and a reduced number of processes (Fig. 5C). M-CSF treatment of $\mathrm{Gba}^{-/} \mathrm{NSCs}$ resulted in increased efficiency of neuronal differentiation, along with an increase in the number of processes and enhanced neuronal outgrowth (Fig. 5C). Taken together, our findings suggest that M-CSF increases selfrenewal, proliferation, neuronal differentiation and neuronal morphogenesis of $\mathrm{Gba}^{-/} \mathrm{NSCs}$.

\section{DISCUSSION}

The present study is a part of a search for novel therapeutic strategies to treat neuronopathic GD, which are based on the concept of neuronal regeneration. Neuropathologically, human and murine GD is characterized by neuronal loss, neuron- phagia, and demyelination (Adachi et al., 1967; Farfel-Becker et al., 2011; Kaga et al., 1982; 1998). These histological changes result in the clinical features observed in patients, including hypertonia of the neck, seizures, and ataxia (Tayebi et al., 1998; Tylki-Szymańska et al., 2010). The principal goal of neuronopathic GD therapy is to restore function to malfunctioning cells, particularly neurons. We observed that the ratio of neuronal differentiation was not significantly different between $\mathrm{Gba}^{-}$ ${ }^{-}$and $\mathrm{Gba}^{+/}$NSCs. However, terminally differentiated neurons from $\mathrm{Gba}^{-/}$NSCs showed reduced neurite outgrowth and number of processes. Therefore, stimulating neurogenesis is appropriate for the treatment of patients with GD.

Several neurogenic activities associated with BM-MSCs have been investigated (Deng et al., 2006; Zhang et al., 2004). Our findings presented here correspond with those from previous investigations regarding the stimulatory effects of BM-MSCs on neurogenesis (Croft and Przyborski, 2009; Lee et al., 2013; Yoo et al., 2008). In vitro observations demonstrated that indirect coculture with BM-MSCs could result in the alleviation of defects associated with the self-renewal and proliferation of $\mathrm{Gba}^{-/}$ NSCs. We also observed that their differentiation into neurons or oligodendrocytes was enhanced. These findings suggest that the effects of BM-MSCs on self-renewal, proliferation and differentiation can be attributed to the indirect effects of soluble factors secreted from BM-MSCs and that these cells selectively promote neurogenesis and oligogenesis from NSCs. The neurogenic effect of BM-MSCs is mediated by a variety of soluble factors (Croft and Przyborski, 2009). Although the differentiation potential of BM-MSCs for tissue repair has been demonstrated numerous times, a repeated finding from several experiments 
A

\begin{tabular}{|c|c|c|c|c|c|c|c|c|c|c|c|c|c|c|}
\hline & A & B & c & D & $\mathrm{E}$ & $\mathrm{F}$ & G & H & 1 & $J$ & $\mathrm{~K}$ & L & M & $\mathrm{N}$ \\
\hline 1 & POS & POS & NEG & NEG & 6Ckine & ACE & bFGF & $\begin{array}{l}\text { Complement } \\
\text { C5a }\end{array}$ & DKK-1 & EGF & Fractalkine & GCSF & GM-CSF & HGF \\
\hline 2 & IFN-gamma & $\begin{array}{c}\text { IFN- } \\
\text { gamma } \\
\mathrm{RI}\end{array}$ & IGFBP-3 & IGF-I & IL-1alpha & IL-1beta & IL-1ra & IL-2 & IL-4 & IL-6 & IL-6 R & IL-10 & IL-12p40/70 & IL-12p70 \\
\hline 3 & IL-13 & IL-17A & IL-20 & IL-23p19 & MCP-1 & M-CSF & MIP-1alpha & MIP-2 & Neprilysin & MMP-2 & MMP-3 & P-Selectin & RAGE & RANTES \\
\hline 4 & SDF-1 & Shh N & TNF RI & TNF RII & TGF-beta1 & TNF-alpha & TRAIL & VCAM-1 & VEGF & VEGF R2 & VEGF R3 & VEGE-D & BLANK & POS \\
\hline
\end{tabular}

$\mathrm{Gba}^{+/-}$

$\mathrm{Gba} /$

$\mathrm{Gba}^{-/}+\mathrm{BM}-\mathrm{MSCS}$
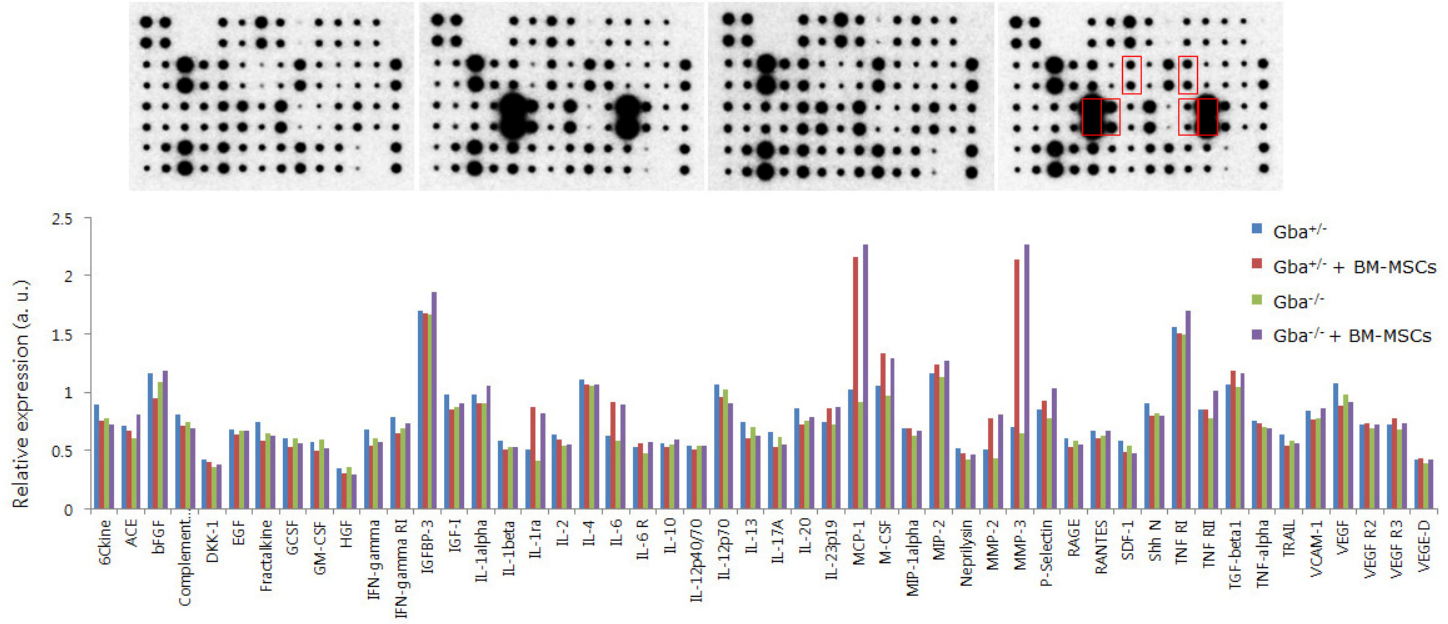

B

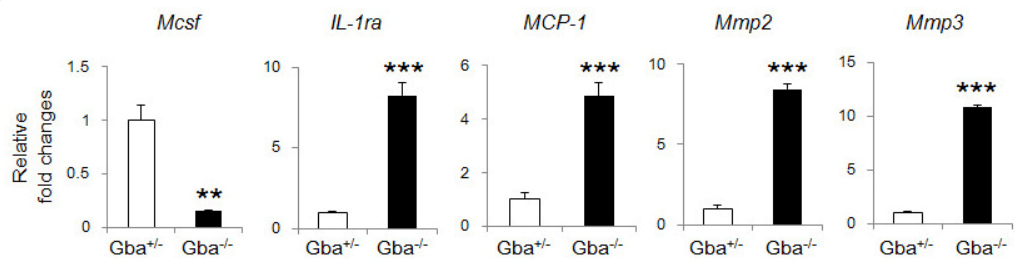

C

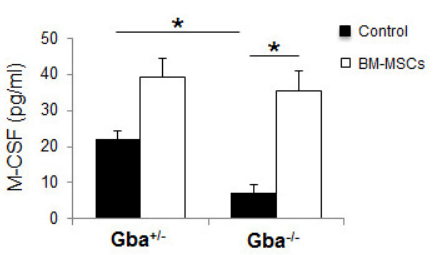

Fig. 4. BM-MSCs increased neurotrophic factor signaling. (A) A cytokine antibody array was incubated with conditioned media recovered from three-dimensional cultures of BM-MSC-treated NSCs or untreated NSCs. Co-culture of Gba ${ }^{-1}$ NSCs with BM-MSCs resulted in the upregulation of M-CSF, IL-1ra, MCP-1, MMP-2, and MMP-3 (red squared spots). Densitometry analyses of cytokine/chemokine signals were normalized to positive and negative antibody array controls for $\mathrm{Gba}^{+/-} \mathrm{NSCs}$. (B) Analysis using qPCR assays revealed greater transcriptional downregulation of M-CSF in NSCs from $\mathrm{Gba}^{-/}$mice compared with that in $\mathrm{Gba}^{+/}$mice ( $n=4$ per group). ${ }^{*} p<0.05$ compared with the $\mathrm{Gba}^{+/}$controls. (C) ELISA analysis was used to assess M-CSF expression in NSCs. M-CSF expression was reduced to a greater extent in NSCs of Gba-mice compared with that in $\mathrm{Gba}^{+/}$NSCs. Gba NSCs co-cultured with BM-MSCs exhibited greater M-CSF expression levels compared with those in $\mathrm{Gba}^{-/-}$NSCs that were not co-cultured with BM-MSCs ( $n=5$ mice per group). All data are presented as the mean \pm SEM. ${ }^{*} p<0.05$.

was that these cells frequently confer functional improvements without significant evidence of either engraftment or differentiation (Parr et al., 2007; Prockop, 2007). Results from these studies suggest that the repair of tissues by BM-MSCs does not occur solely as a result of their stem cell-like ability to differentiate, but could also be attributed to the release of growth factors and other molecules that might elicit therapeutic effects (Prockop et al., 2003). To determine the paracrine factors that mediate MSC effects on the proliferation of $\mathrm{Gba}^{-/-} \mathrm{NSCs}$, we used cytokine arrays for analysis of media samples from cocultures. Our results revealed increased expression of M-CSF in the conditioned media of BM-MSC co-cultures. We also observed that the effects of BM-MSCs on proliferation and differentiation were increased for $\mathrm{Gba}^{-/-} \mathrm{NSC}$ cultures treated with M-CSF. Previous research has shown that, independent of neuropathology, chemokines and their receptors are expressed in the developing brain, and play roles in orienting cell migration trophic support, proliferation, and/or differentiation (Bajetto et al., 2001). The M-CSF receptor (CSF-1R) is expressed in neural progenitors and plays a direct role in the regulation of progenitor cell proliferation and differentiation (Nandi et al., 2012). The direct regulation of neural progenitors through CSF-1R is biologically significant as demonstrated by the perinatal lethality of Csf1 floxflox $_{\text {, nestin-Cre mice, which phenocopies the perinatal }}$ death of Csf1r-- mice (Nandi et al., 2012). These results suggest that M-CSF is an effective adjuvant for improving neurogenic effects, and that M-CSF derived from BM-MSCs could be a critical factor for increasing the proliferation and neural differentiation of $\mathrm{Gba}^{--}$NSCs.

In conclusion, our data suggest that BM-MSCs can improve the developmental deficits of $\mathrm{Gba}^{- \text {- }} \mathrm{NSC}$ s by augmenting proliferation, differentiation, and neuronal morphogenesis. We have 


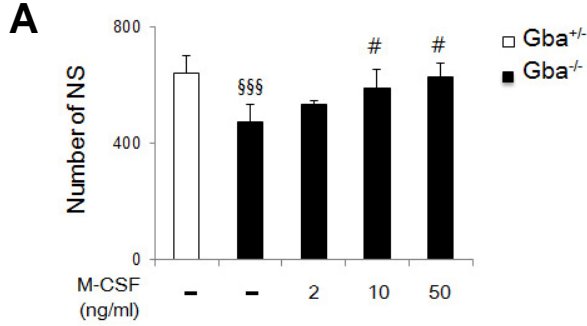

B

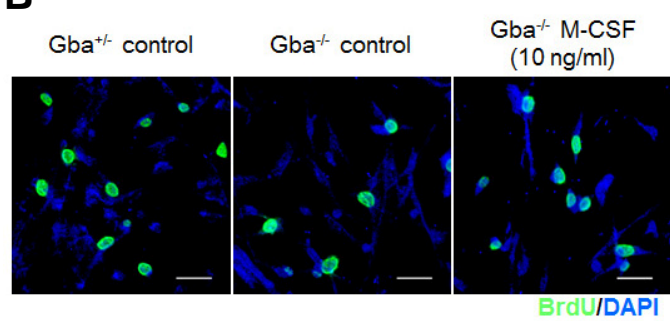

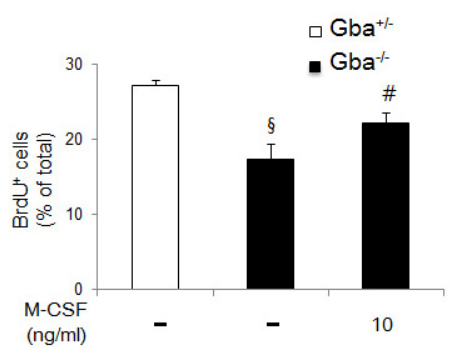

(ng/ml)
Fig. 5. M-CSF enhances the self-renewal, proliferation, and neuronal differentiation of $\mathrm{Gba}^{--}$NSCs. (A) Gba ${ }^{--}$NSCs were treated with different concentrations of recombinant murine M-CSF $(2-50 \mathrm{ng} / \mathrm{ml})$, and selfrenewal was assessed. M-CSF at concentrations of 10 and $50 \mathrm{ng} / \mathrm{ml}$ increased the number of $\mathrm{Gba}^{-\alpha} \mathrm{NSs}$ ( $n=3$ per group). (B) M-CSF affects the proliferation of $\mathrm{Gba}^{-/-} \mathrm{NSCs}$ (scale bar, $20 \mu \mathrm{m}$ ). Proliferation ability was assessed by the percentage of BrdU-positive cells ( $n=3$ per group). (C) Representative fluorescence images and quantitation of $\beta$ III-tubulin (scale bar, $50 \mu \mathrm{m} ; n=3$ per group). All data are presented as the mean \pm SEM. ${ }^{\S} p<0.05,{ }^{\S \S} p<0.005$ compared with $\mathrm{Gba}^{+/}$controls. ${ }^{*} p<0.05$ compared with non-treated controls. ${ }^{*} p<0.05,{ }^{* * *} p<$ 0.005 .

C
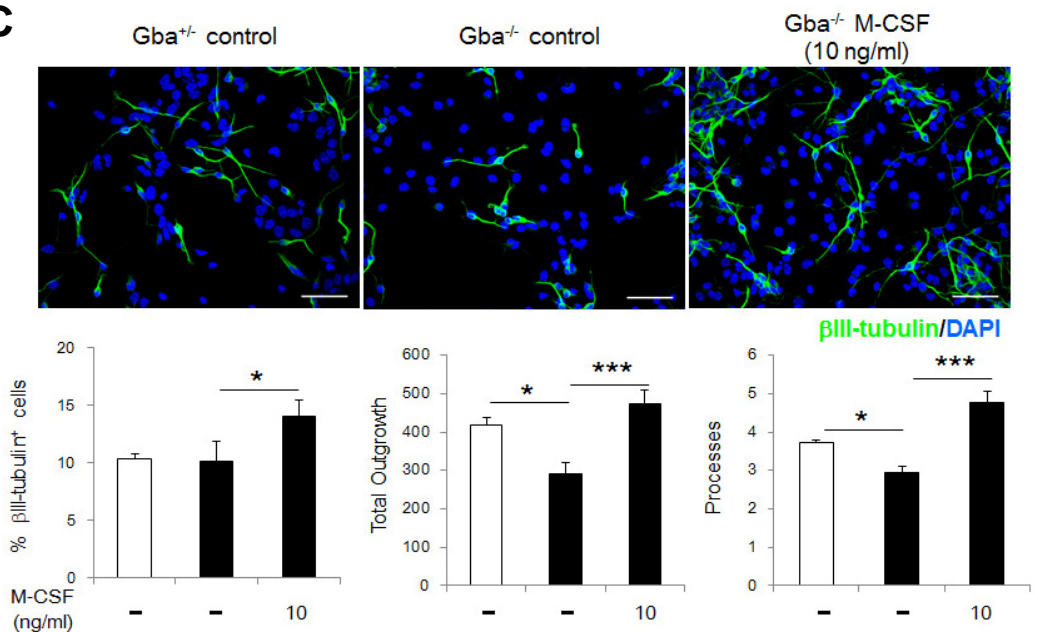

provided evidence that BM-MSCs can act as key mediators of neurogenesis, which could in turn have implications for the management of GD.

\section{ACKNOWLEDGMENTS}

This research was supported by a grant of the Korea Health Technology R\&D Project through the Korea Health Industry Development Institute (KHIDI), funded by the Ministry of Health \& Welfare, Republic of Korea (HI12C0313).

\section{REFERENCES}

Adachi, M., Wallace, B.J., Schneck, L., and Volk, B.W. (1967). Fine structure of central nervous system in early infantile Gaucher's disease. Arch. Pathol. 83, 513-526.

Bae, J.S., Han, H.S., Youn, D.H., Carter, J.E., Modo. M., Schuchman, E.H., and Jin, H.K. (2007). Bone marrow-derived mesenchymal stem cells promote neuronal networks with functional synaptic transmission after transplantation into mice with neurodegeneration. Stem Cells 25, 1307-1316.

Bajetto, A., Bonavia, R., Barbero, S., Florio, T., and Schettini, G. (2001). Chemokines and their receptors in the central nervous system. Front Neuroendocrinol. 22, 147-184.
Beutler, E., and Grabowski, G.A. (2001). Glucosylceramide lipidosisGaucher disease. In: C.R. Scriver, A.L. Beaudet, W.S. Sly, D. Valle, eds. The metabolic and molecular bases of inherited diseases. 8th ed. (New York, USA: McGraw-Hill), pp. 3635-3668.

Block, G.J., Ohkouchi, S., Fung, F., Frenkel, J., Gregory, C., Pochampally, R., DiMattia, G., Sullivan, D.E., and Prockop, D.J. (2009). Multipotent stromal cells are activated to reduce apoptosis in part by upregulation and secretion of stanniocalcin-1. Stem Cells 27, 670-681.

Brady, R.O., Kanfer, J.N., Bradley, R.M., and Shapiro, D. (1966). Demonstration of a deficiency of glucocerebroside-cleaving enzyme in Gaucher's disease. J. Clin. Invest. 45, 1112-1115.

Chen, Y., Balasubramaniyan, V., Peng, J., Hurlock, E.C., Tallquist, M., Li, J., and Lu, Q.R. (2007). Isolation and culture of rat and mouse oligodendrocyte precursor cells. Nat. Protoc. 2, 10441051.

Conradi, N.G., Sourander, P., Nilsson, O., Svennerholm, L., and Erikson, A. (1984). Neuropathology of the Norrbottnian type of Gaucher disease. Morphological and biochemical studies. Acta Neuropathol. 65, 99-109.

Conradi, N.G., Kalimo, H., and Sourander, P. (1988). Reactions of vessel walls and brain parenchyma to the accumulation of Gaucher cells in the Norrbottnian type (type III) of Gaucher disease. Acta Neuropathol. 75, 385-390.

Croft, A.P., and Przyborski, S.A. (2009). Mesenchymal stem cells 
expressing neural antigens instruct a neurogenic cell fate on neural stem cells. Exp. Neurol. 216, 329-341.

Deng, Y.B., Liu, X.G., Liu, Z.G., Liu, X.L., Liu, Y., and Zhou, G.Q. (2006). Implantation of BM mesenchymal stem cells into injured spinal cord elicits de novo neurogenesis and functional recovery: evidence from a study in rhesus monkeys. Cytotherapy 8 , 210-214.

Enquist, I.B., Lo Bianco, C., Ooka, A., Nilsson, E., Månsson, J.E., Ehinger, M., Richter, J., Brady, R.O., Kirik, D., Karlsson, S. (2007). Murine models of acute neuronopathic Gaucher disease. Proc. Natl. Acad. Sci. USA 104, 17483-17488.

Farfel-Becker, T., Vitner, E., Dekel, H., Leshem, N., Enquist, I.B., Karlsson, S., and Futerman, A.H. (2009). No evidence for activation of the unfolded protein response in neuronopathic models of Gaucher disease. Hum. Mol. Genet. 18, 1482-1488.

Farfel-Becker, T., Vitner, E.B., Pressey, S.N., Eilam, R., Cooper, J.D. Futerman, A.H. (2011). Spatial and temporal correlation between neuron loss and neuroinflammation in a mouse model of neuronopathic Gaucher disease. Hum. Mol..Genet. 20, 1375-1386.

He, X., Dagan, A., Gatt, S., and Schuchman, E.H. (2005). Simultaneous quantitative analysis of ceramide and sphingosine in mouse blood by naphthalene-2,3-dicarboxyaldehyde derivatization after hydrolysis with ceramidase. Anal. Biochem. 340, 113-122.

He, X., Huang, Y., Li, B., Gong, C.X., and Schuchman, E.H. (2010). Deregulation of sphingolipid metabolism in Alzheimer's disease. Neurobiol. Aging 31, 398-408.

Kaga, M., Azuma, C., Imamura, T., Murakami, T., and Kaga, K. (1982). Auditory brainstem response (ABR) in infantile Gaucher's disease. Neuropediatrics 13, 207-210.

Kaga, K., Ono, M., Yakumaru, K., Owada, M., and Mizutani, T. (1998). Brainstem pathology of infantile Gaucher's disease with only wave I and II of auditory brainstem response. J. Laryngol. Otol. 112, 1069-1073.

Lee, H., Kang, J.E., Lee, J.K., Bae, J.S., and Jin, H.K. (2013). Bone-marrow-derived mesenchymal stem cells promote proliferation and neuronal differentiation of Niemann-Pick type C mouse neural stem cells by upregulation and secretion of CCL2. Hum. Gene. Ther. 24, 655-669.

Ming, G.L., and Song, H. (2011). Adult neurogenesis in the mammalian brain: significant answers and significant questions. Neuron 70, 687-702.

Motabar, O., Goldin, E., Leister, W., Liu, K., Southall, N., Huang, W., Marugan, J.J., Sidransky, E., and Zheng, W. (2012). A high throughput glucocerebrosidase assay using the natural substrate glucosylceramide. Anal. Bioanal. Chem. 402, 731-739.
Nandi, S., Gokhan, S., Dai, X.M., Wei, S., Enikolopov, G., Lin, H., Mehler, M.F., and Stanley, E.R. (2012). The CSF-1 receptor ligands IL-34 and CSF-1 exhibit distinct developmental brain expression patterns and regulate neural progenitor cell maintenance and maturation. Dev. Biol. 367, 100-113.

Parr, A.M., Tator, C.H., and Keating, A. (2007). Bone marrow derived mesenchymal stromal cells for the repair of central nervous system injury. Bone Marrow Transplant. 40, 609-619.

Prockop, D.J. (2007). "Stemness" does not explain the repair of many tissues by mesenchymal stem/multipotent stromal cells (MSCs). Clin. Pharmacol. Ther. 82, 241-243.

Prockop, D.J., Gregory, C.A., and Spees, J.L. (2003). One strategy for cell and gene therapy: harnessing the power of adult stem cells to repair tissues. Proc. Natl. Acad. Sci. USA 100, $11917-$ 11923.

Ren, G., Li, T., Lan, J.Q., Wilz, A., Simon, R.P., and Boison, D. (2007). Lentiviral RNAi-induced downregulation of adenosine kinase in human mesenchymal stem cell grafts: a novel perspective for seizure control. Exp. Neurol. 208, 26-37.

Reynolds, B.A., and Weiss, S. (1996). Clonal and population analyses demonstrate that an EGF-responsive mammalian embryonic CNS precursor is a stem cell. Dev. Biol. 175, 1-13.

Sidransky, E. (2004). Gaucher disease: complexity in a 'simple' disorder. Mol. Genet. Metab. 83, 6-15.

Tayebi, N., Reissner, K.J., Lau, E.K., Stubblefield, B.K., Klineburgess, A.C., Martin, B.M., and Sidransky, E. (1998). Genotypic heterogeneity and phenotypic variation among patients with type 2 Gaucher's disease. Pediatr. Res. 43, 571-578.

Tylki-Szymanska, A., Vellodi, A., El-Beshlawy, A., Cole, J.A., and Kolodny, E. (2010). Neuronopathic Gaucher disease: demographic and clinical features of 131 patients enrolled in the International Collaborative Gaucher Group Neurological Outcomes Subregistry. J. Inherit. Metab. Dis. 33, 339-346.

Yoo, S.W., Kim, S.S., Lee, S.Y., Lee, H.S., Kim, H.S., Lee, Y.D. Suh-Kim, H. (2008). Mesenchymal stem cells promote proliferation of endogenous neural stem cells and survival of newborn cells in a rat stroke model. Exp. Mol. Med. 40, 387-397.

Zhang, J., Li, Y., Chen, J., Yang, M., Katakowski, M., Lu, M., and Chopp, M. (2004). Expression of insulin-like growth factor 1 and receptor in ischemic rats treated with human marrow stromal cells. Brain Res. 1030, 19-27.

Zhang, J., Li, Y., Chen, J., Cui, Y., Lu, M., Elias, S.B., Mitchell, J.B., Hammill, L., Vanguri, P, and Chopp, M. (2005). Human bone marrow stromal cell treatment improves neurological functional recovery in EAE mice. Exp. Neurol. 195, 16-26. 